\title{
MEKK1, MKK1/MKK2 and MPK4 function together in a mi- togen-activated protein kinase cascade to regulate innate immunity in plants
}

\author{
Minghui Gao ${ }^{1,2,{ }^{*}}$, Jinman Liu ${ }^{1,2,{ }^{*}}$, Dongling $\mathrm{Bi}^{2}$, Zhibin Zhang ${ }^{2}$, Fang Cheng ${ }^{2}$, Sanfeng Chen $^{3}$, Yuelin Zhang $^{2}$

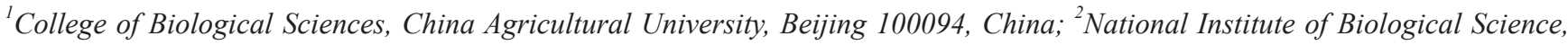 \\ Zhongguancun Life Science Park, 7 Science Park Road, Beijing 102206, China; ' State Key Laboratories for AgroBiotechnology, \\ China Agricultural University, Beijing 100094, China
}

Mitogen-activated protein kinase (MAPK) cascades play important roles in regulating plant innate immune responses. In a genetic screen to search for mutants with constitutive defense responses, we identified multiple alleles of $m p k 4$ and mekk1 that exhibit cell death and constitutive defense responses. Bimolecular fluorescence complementation (BiFC) analysis showed that both MPK4 and MEKK1 interact with MKK1 and MKK2, two closely related MAPK kinases. $m k k 1$ and $m k k 2$ single mutant plants do not have obvious mutant phenotypes. To test whether MKK1 and MKK2 function redundantly, $m k k 1 m k k 2$ double mutants were generated. The $m k k 1 m k k 2$ double mutant plants die at seedling stage and the seedling-lethality phenotype is temperature-dependent. Similar to the mpk4 and mekk1 mutants, the $m k k 1 m k k 2$ double mutant seedlings accumulate high levels of $\mathrm{H}_{2} \mathrm{O}_{2}$, display spontaneous cell death, constitutively express $\underline{P}$ athogenesis $\underline{R}$ elated $(P R)$ genes and exhibit pathogen resistance. In addition, activation of MPK4 by flg22 is impaired in the $m k k 1 m k k 2$ double mutants, suggesting that MKK1 and MKK2 function together with MPK4 and MEKK1 in a MAP kinase cascade to negatively regulate innate immune responses in plants.

Keywords: MAPK, innate immunity, MPK4, MEKK1, MKK1, MKK2

Cell Research (2008) 18:1190-1198. doi: 10.1038/cr.2008.300; published online 4 November 2008

\section{Introduction}

Eukaryotes use different receptors to recognize microbial pathogens [1]. There are two types of immune receptors in plants. One type recognizes microbe-associated molecular patterns (MAMPs) and initiates downstream immune responses. For example, Arabidopsis FLS2 is required for the recognition of bacterial flagellin and EFR is required for the recognition of bacterial elongation factor EF-Tu $[2,3]$. Both FLS2 and EFR belong to the leucine-rich-repeat (LRR)-containing receptor-like kinase family. The other type of immune receptors is the plant resistance $(\mathrm{R})$ proteins. They are thought to recog-

\footnotetext{
*These two authors contributed equally to this work.

Correspondence: Yuelin Zhang

Tel: +86-010-80726688-8360; Fax: +86-010-80728646

E-mail: zhangyuelin@nibs.ac.cn

Received 24 July 2008; revised 1 September 2008; accepted 10 September 2008; published online 4 November 2008
}

nize effector proteins that are delivered by pathogens to suppress MAMP-triggered immune responses. Compared with MAMP-triggered immune responses, R protein-mediated resistance responses are generally much stronger and often lead to hypersensitive response (HR), a localized programmed cell death event that restricts pathogen growth.

In Arabidopsis, several mutations in $R$ genes have been identified that lead to constitutively activated downstream immune responses. Constitutive activation of these immune receptors has detrimental effects on plant growth and development. ssi4, slh1 and sncl all contain mutations in TIR-NB-LRR-type $R$ genes [4-6]. Both ssi4 and $\operatorname{slh} 1$ mutants show spontaneous cell death. Although sncl mutant plants do not show cell death phenotypes, they accumulate high levels of salicylic acid (SA) and have reduced stature. Recently, a semi-dominant mutant $u n i-1 D$ was found to contain a gain-of-function mutation in CC-NB-LRR-type $R$ genes [7]. The uni-1D mutation causes constitutive activation of defense responses and 
morphological defects through accumulation of abnormal levels of cytokinin. Constitutive activation of MAMP receptor FLS2 by growing plants on medium containing flg22 (a peptide derived from Escherichia coli flagellin) also results in inhibition of plant growth [2]. To prevent the detrimental effects caused by activation of defense responses, plant immune responses must be under tight negative regulations in the absence of pathogen infection.

Activation of mitogen-activated protein kinase (MAPK) cascades is a common mechanism for regulating innate immune responses in both animals and plants [8]. Ara- bidopsis MPK4 is an important negative regulator of systemic acquired resistance (SAR). mpk4 mutant plants in Landsberg ecotype (Ler) accumulate high levels of SA and constitutively express $\underline{\text { Pathogenesis }}$ Related $(P R)$ genes and exhibit pathogen resistance, but have no necrotic lesions [9]. Arabidopsis MEKK1, a MAPK kinase kinase, also functions as a negative regulator of plant immune responses. Knockout mutants of MEKK1 constitutively express $P R$ genes and exhibit a seedling-lethality phenotype because of spontaneous cell death [10-12].

To search for additional negative regulators of plant innate immune response, we looked for mutants that con-
A

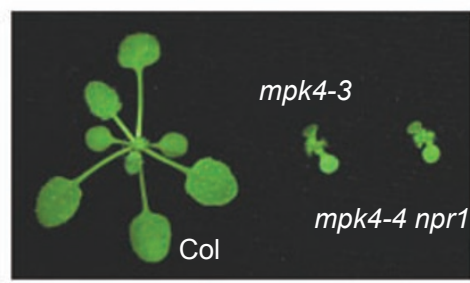

C

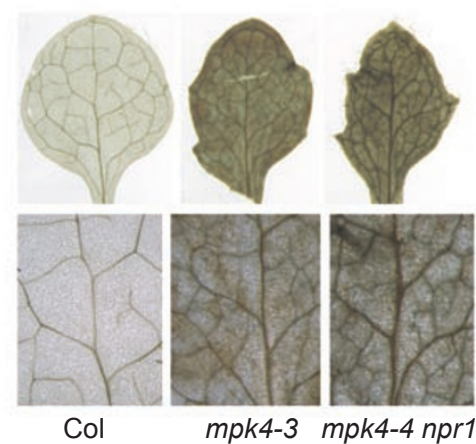

B

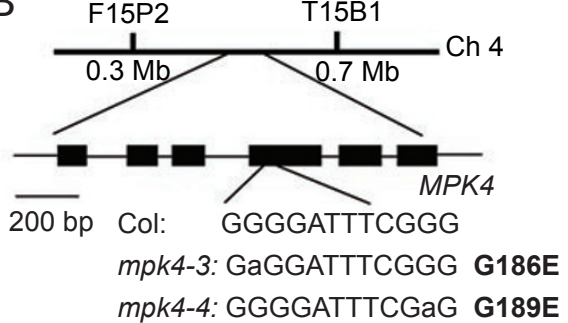

$\mathrm{D}$

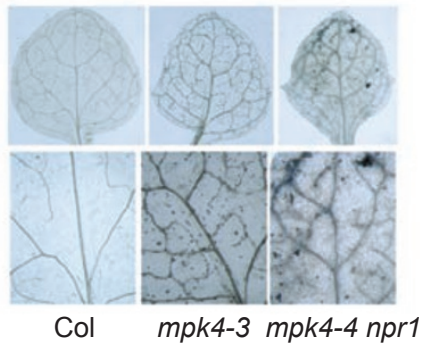

G

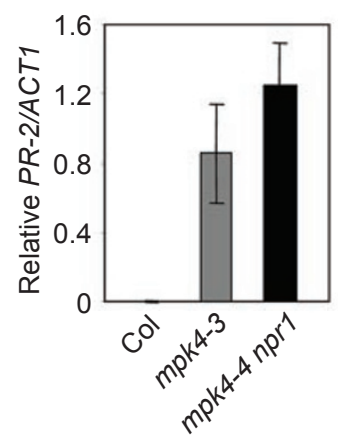

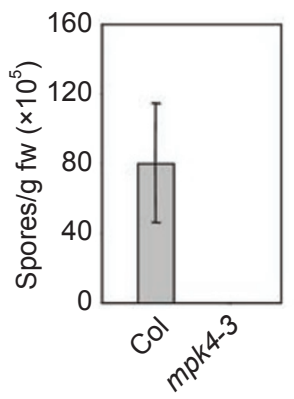

Figure 1 Analysis of mpk4-3 and mpk4-4 npr1-1 mutants. (A) Phenotypes of wild-type, mpk4-3 and mpk4-4 npr1-1. Plants were grown on soil and photographed 3 weeks after planting. (B) Map position and mutations in $m p k 4-3$ and $m p k 4-4$. (C) DAB staining of wild-type, mpk4-3 and mpk4-4 npr1-1 mutant seedlings. (D) Trypan blue staining of wild-type, mpk4-3 and mpk4-4 npr1-1 mutant seedlings. PR-1 (E) and PR-2 (F) expression in the wild-type, mpk4-3 and mpk4-4 npr1-1 mutant seedlings. Error bars represent standard deviation from three measurements. Values were normalized to the expression of ACTIN1. (G) Growth of H. parasitica Noco2 on wild type and mpk4-3. Three-week-old seedlings were sprayed with $H$. parasitica Noco2 spores $\left(10^{5}\right.$ spores $\left./ \mathrm{ml}\right)$. Infection was scored 7 days after inoculation by counting the number of conidia spores per gram of leaf samples. Error bars represent standard deviation from three measurements. 
stitutively express $P R$ genes and exhibit pathogen resistance. We screened an EMS-mutagenized mutant population and identified multiple mutant alleles of $m p k 4$ and $m e k k 1$. Unlike the original $m p k 4$ mutant identified in Ler background, the $m p k 4$ mutant alleles we obtained in the Columbia background were more like the mekkl knockout mutants and died at seedling stage because of spontaneous cell death. We found that both MPK4 and MEKK1 interact with two MAPK kinases, MKK1 and MKK2, in vivo, and $m k k 1 m k k 2$ double mutant plants exhibit very similar phenotypes to mekkl and mpk4 mutants. Our results suggest that a MAP kinase cascade consisting of MEKK1, MKK1/MKK2 and MPK4 negatively regulates plant innate immunity.

\section{Results}

\section{Identification and characterization of alleles of mpk4}

To identify mutants that are constitutively resistant to pathogens, progeny from individual EMS-mutagenized M1 mutant lines in Columbia background were analyzed for resistance to Hyaloperonospora parasitica Noco2 ( $H$. $p$. Noco2). One of the mutants resistant to H. $p$. Noco2 was found to die at the seedling stage (Figure 1A). We mapped the mutation to a $400-\mathrm{kb}$ region on chromosome IV, where MPK4 is localized. Sequence analysis revealed a point mutation in the mutant; thus we named it mpk43 (Figure 1B). 3, 3'-Diaminobenzidine (DAB) staining showed that the mpk4-3 plants also accumulated high levels of $\mathrm{H}_{2} \mathrm{O}_{2}$ (Figure 1C). Trypan blue staining showed that there was extensive cell death in the mpk4-3 mutant (Figure 1D). Both $P R-1$ and $P R-2$ are constitutively expressed in the mpk4-3 mutant (Figure $1 \mathrm{E}$ and $1 \mathrm{~F}$ ). In addition, the mpk4-3 mutant plants are constitutively resistant to H. $p$. Noco2 (Figure 1G).

We also identified another allele of mpk4 (mpk4-4) in a suppressor screen of nprl-1 (nonexpresser of $P R$ genes 1,1$)$ to look for mutants that constitutively express the BGL2-GUS reporter gene in the npr 1-1 mutant background. The npr 1-1 mutation blocks SA-induced $P R$ gene expression and the $B G L 2-G U S$ reporter gene expression. Like $m p k 4-3$, the $m p k 4-4$ mutant accumulates high levels of $\mathrm{H}_{2} \mathrm{O}_{2}$, shows extensive cell death and exhibits seedling-lethality phenotypes (Figure 1A, $1 \mathrm{C}$ and $1 \mathrm{D})$. Whereas $P R-2$ is constitutively expressed in the mpk4-4 npr 1-1 mutant plants, $P R-1$ expression is blocked by the nprl-1 mutation (Figure $1 \mathrm{E}$ and $1 \mathrm{~F}$ ). Through similar mapping and targeted gene-sequencing approaches, two other mutants that constitutively express the BGL2-GUS reporter gene in the npr 1-1 mutant background and exhibit seedling-lethality phenotypes were later found to be alleles of mekkl (Figure 2). Interesting-
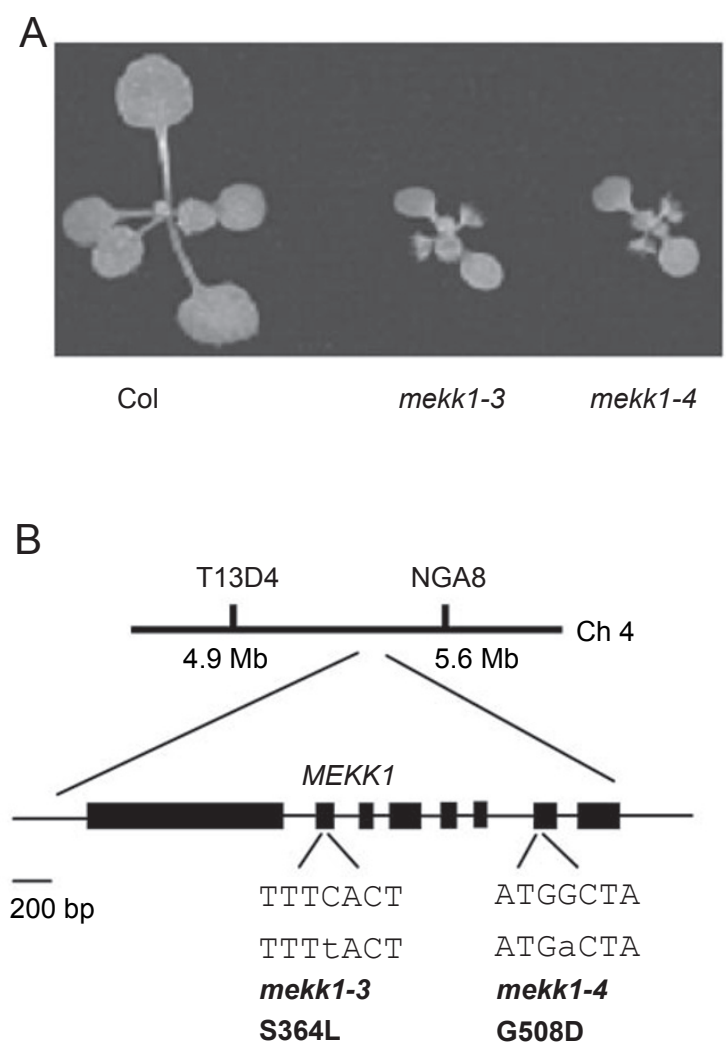

Figure 2 Analysis of mekk1-3 and mekk1-4 mutants. (A) Phenotypes of wild type, mekk1-3 and mekk1-4. Plants were grown on soil and photographed 3 weeks after planting. (B) Map position and mutations in mekk1-3 and mekk1-4.

ly, the seedling-lethality phenotypes of $m p k 4$ and $m e k k 1$ alleles are temperature dependent (observed at $22^{\circ} \mathrm{C}$ ). At $28{ }^{\circ} \mathrm{C}$, these mutant plants can complete their life cycles and set seeds.

\section{MKK1 and MKK2 interact with MPK4 and MEKK1 in} vivo

MKK1 and MKK2 are two closely related MAP kinase kinases that interact with MPK4 and MEKK1 in yeast two-hybrid assays [13-15]. To test whether MKK1 and MKK2 associate with MPK4 and MEKK1 in vivo, we analyzed their interactions using a bimolecular fluorescence complementation (BiFC) approach [16]. MKK1 and MKK2 were fused to the N-terminal fragment of YFP $\left(\right.$ MKK1-YFP ${ }^{\mathrm{N}}$ and MKK2-YFP ${ }^{\mathrm{N}}$ ), whereas MEKK1 and MPK4 were fused to the C-terminal fragment of YFP $\left(\mathrm{MEKK} 1-\mathrm{YFP}^{\mathrm{C}}\right.$ and MPK4-YFP ${ }^{\mathrm{C}}$ ). If two proteins associate with each other, a fluorescent YFP complex would be formed. As shown in Figure 3, YFP fluorescence was observed on the plasma membrane of Arabidopsis mesophyll protoplasts co-transformed with the MKK1-YFP 
and MEKK1-YFP ${ }^{\mathrm{C}}$ constructs. Similar results were observed when MKK2-YFP ${ }^{\mathrm{N}}$ and MEKK1-YFP ${ }^{\mathrm{C}}$ constructs were co-transformed into the Arabidopsis mesophyll protoplasts, suggesting that MKK1 and MKK2 interact with MEKK1 on the plasma membrane. As negative controls, MKK1-YFP ${ }^{\mathrm{N}}$ or MKK2-YFP ${ }^{\mathrm{N}}$ was co-transformed with the $\mathrm{YFP}^{\mathrm{C}}$ vector and no YFP fluorescence was observed. Co-transformation of MEKK1-YFP ${ }^{\mathrm{C}}$ and the $\mathrm{YFP}^{\mathrm{N}}$ vector into protoplasts did not result in YFP fluorescence either.

In contrast, YFP fluorescence was observed on the plasma membrane and in the nucleus of Arabidopsis mesophyll protoplasts co-transformed with the MPK4-YFP and MKK1-YFP ${ }^{\mathrm{N}}$ or MKK2-YFP ${ }^{\mathrm{N}}$ constructs (Figure 3), suggesting that MPK4 interacts with MKK1 and MKK2 on the plasma membrane and in the nucleus. As negative controls, MPK4-YFP ${ }^{\mathrm{C}}$ was transformed into protoplasts

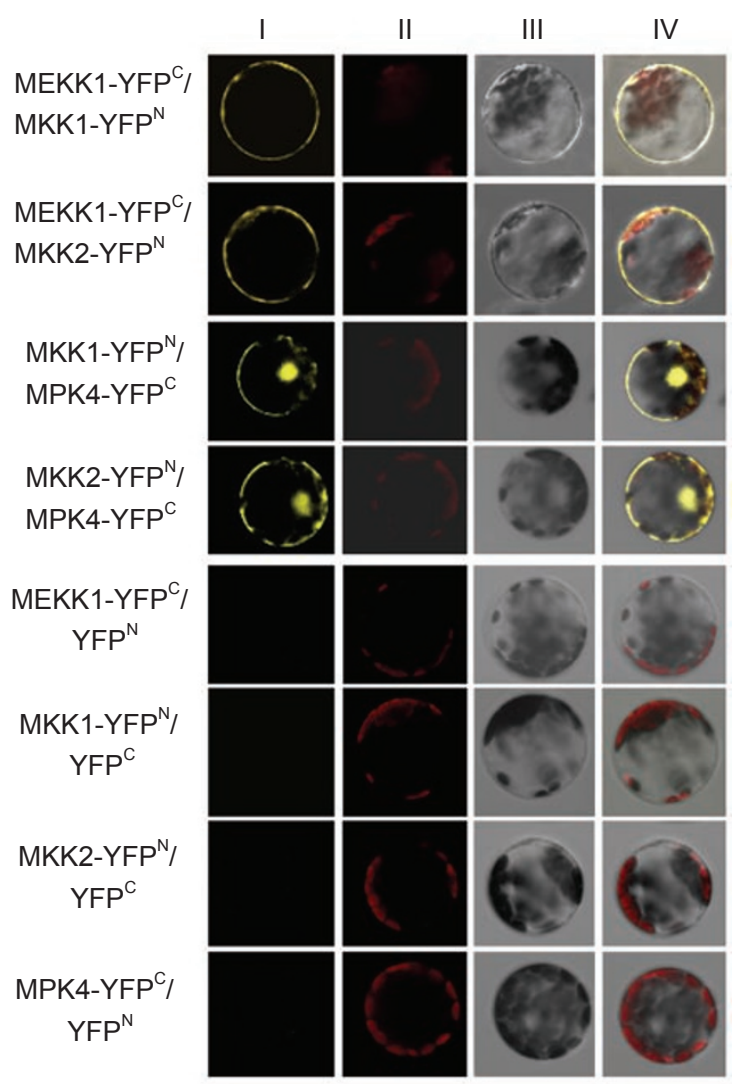

Figure 3 Analysis of MKK1 and MKK2 interactions with MEKK1 and MPK4 by BiFC. Epifluorescence (I), chloroplast autofluorescence (II), bright field (III) and merged (IV) images of Arabidopsis mesophyll protoplasts co-transfected with constructs expressing different fusion proteins (top four panels). Vectors expressing the $\mathrm{N}$ - or $\mathrm{C}$-terminal domains of YFP alone with each corresponding fusion construct were used as negative controls (lower four panels).
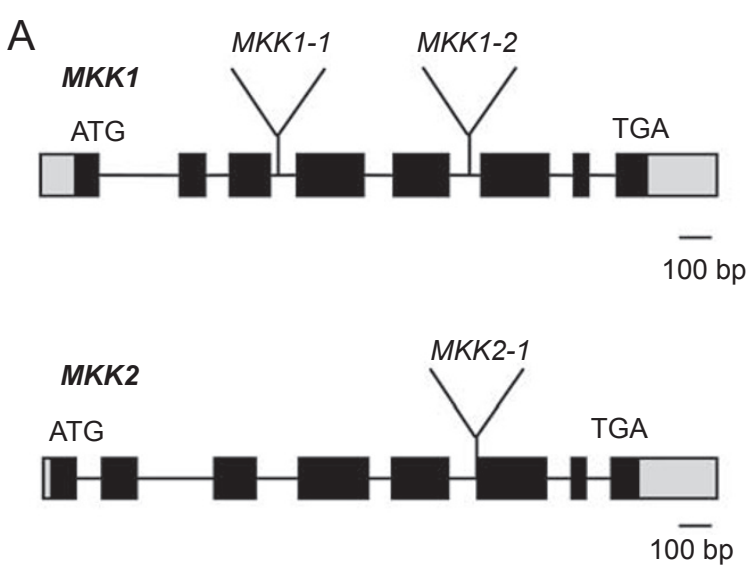

B

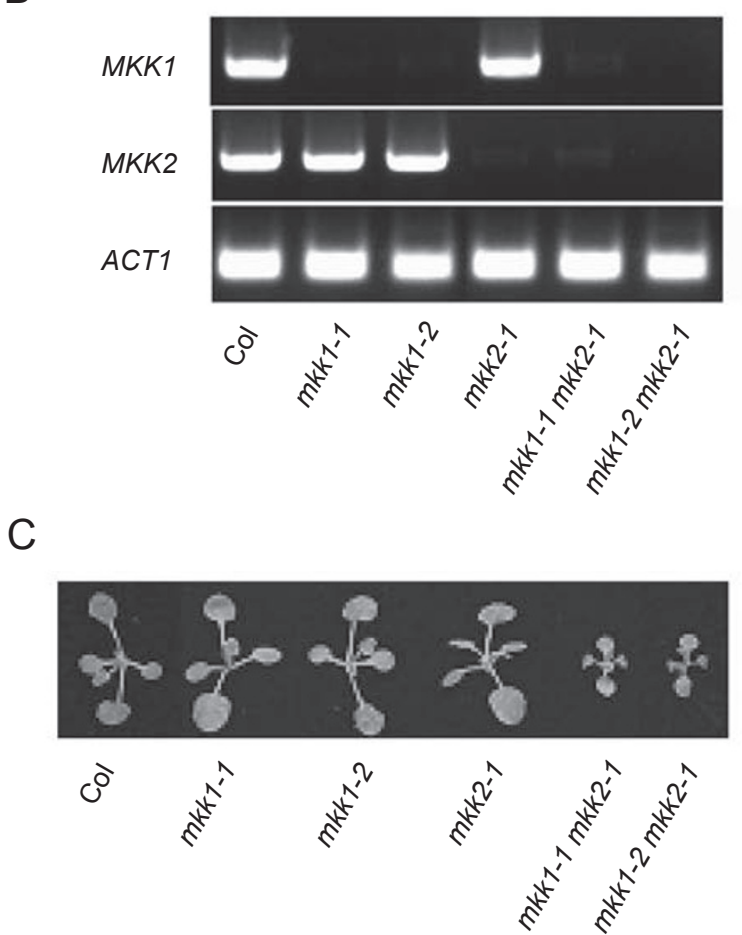

Figure 4 Analysis of the $m k k 1$ and $m k k 2$ insertion mutants. (A) Positions of T-DNA insertions within the MKK1 and MKK2 genes in $m k k 1$ and $m k k 2$ mutants. (B) RT-PCR analysis of MKK1 and MKK2 expression in the knockout mutants. (C) Phenotypes of wild-type, mkk1, mkk2 and mkk1 mkk2 double mutants. Plants were grown on soil and photographed 3 weeks after planting.

together with the $\mathrm{YFP}^{\mathrm{N}}$ vector and no YFP fluorescence was observed.

mkk1 mkk2 double mutant plants exhibit lethality at seedling stage

To test whether MKK1 and MKK2 have similar functions as MEKK1 and MPK4 in regulating cell death and 
A

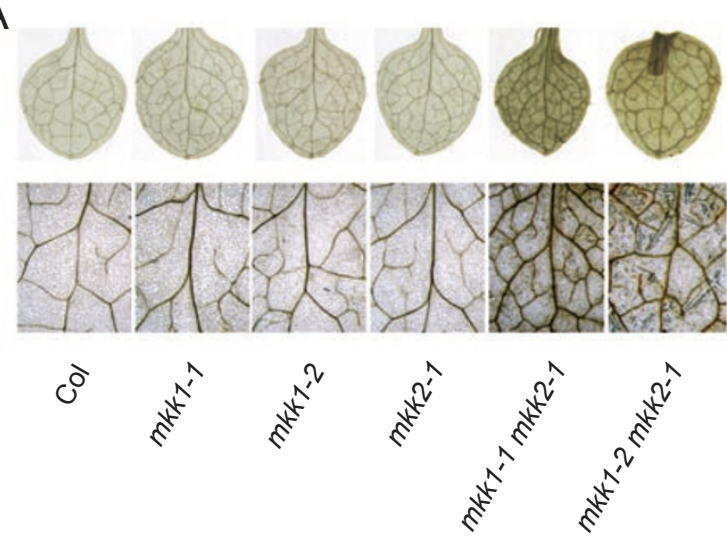

B

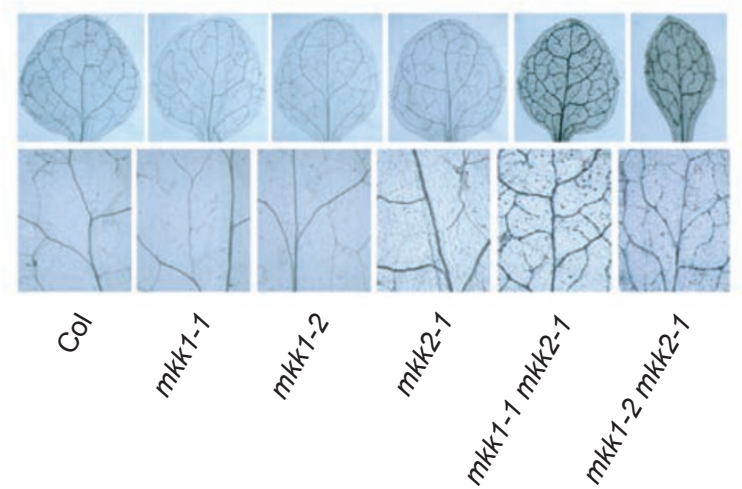

Figure $5 \mathrm{DAB}$ staining (A) and trypan blue staining (B) of wildtype, $m k k 1, m k k 2$ and $m k k 1 m k k 2$ double mutant seedlings. defense responses, T-DNA insertion knockout mutants of $M K K 1$ and $M K K 2$ were obtained from ABRC. The locations of the T-DNA insertion are shown in Figure 4A. RT-PCR analysis of MKK1 and MKK2 in the knockout mutant indicates that expression of the target genes is abolished by the T-DNA insertions (Figure 4B). As MKK1 and MKK2 have high sequence similarity and single knockout mutants do not exhibit any obvious mutant phenotypes, $m k k 1 m k k 2$ double mutants were obtained by crossing the single mutants. As shown in Figure 4C, $m k k 1-1 m k k 2-1$ and $m k k 1-2 m k k 2-1$ double mutant plants are dwarf and small. Similar to the $m p k 4$ and mekk1 mutants, $m k k 1 m k k 2$ double mutant plants exhibit a seedling-lethality phenotype.

As only one $m k k 2$ allele was identified, we tested whether the morphological phenotypes observed in the mkk1-1 mkk2-1 double mutant co-segregate with the $m k k 2-1$ mutation. The progeny of F2 lines that are heterozygous for the $m k k 2-1$ mutation and homozygous for the $m k k 1-1$ mutation were planted. Among 160 plants, 36 displayed $m k k 1 m k k 2$ double mutant morphology. PCR analysis showed that all 36 plants were homozygous for the $m k k 2-1$ mutation, indicating that the morphological phenotypes observed in the mkk1-1 mkk2-1 double mutant co-segregate with the $m k k 2-1$ mutation.

Analysis of cell death and $\mathrm{H}_{2} \mathrm{O}_{2}$ accumulation in mkkI mkk2 plants

To test whether cell death occurred in the $m k k 1 m k k 2$
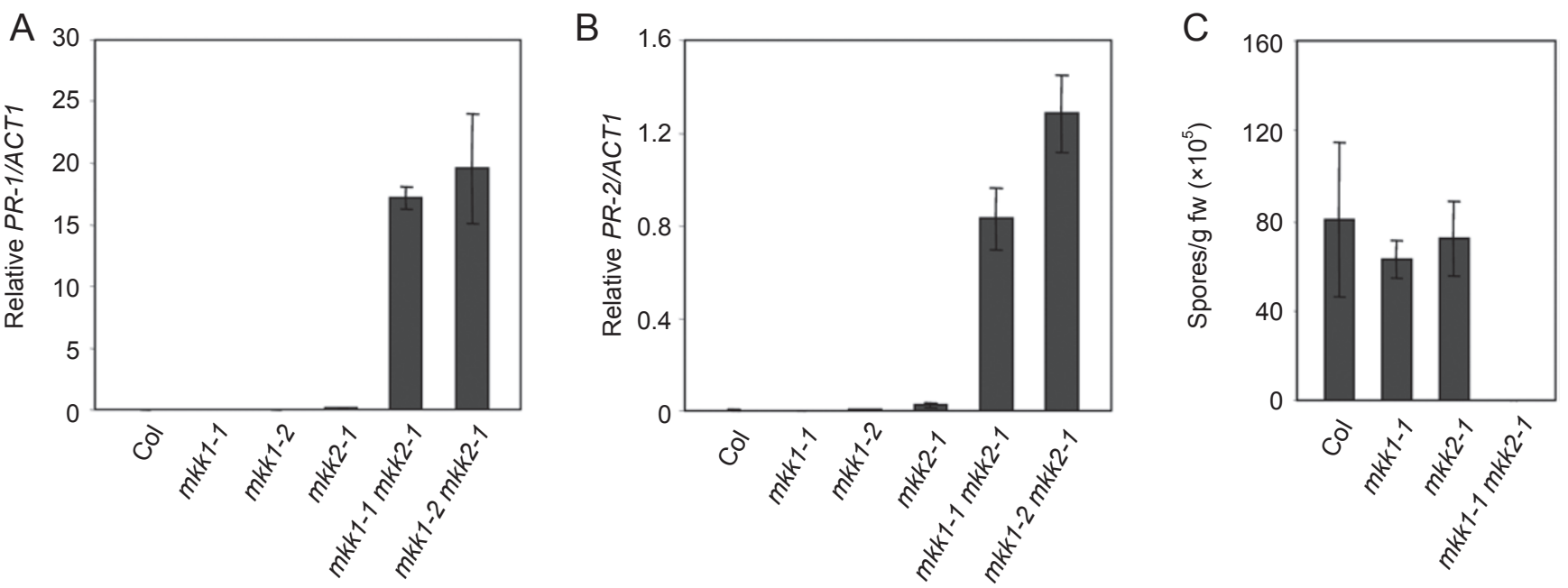

Figure 6 Characterization of the $m k k 1 m k k 2$ double mutant plants. $P R-1(\mathbf{A})$ and $P R-2(\mathbf{B})$ expression in wild-type, $m k k 1$, $m k k 2$ and $m k k 1$ mkk2 double mutants. Error bars represent standard deviations from three measurements. Values were normalized to the expression of ACTIN1. (C) Growth of $H$. parasitica Noco2 on wild-type, mkk1, mkk2 and $m k k 1 m k k 2$ double mutants. Three-week-old seedlings were sprayed with $H$. parasitica Noco2 spores $\left(10^{5} \mathrm{spores} / \mathrm{ml}\right)$. Infection was scored 7 days after inoculation by counting the number of conidia spores per gram of leaf samples. Error bars represent standard deviations from three measurements. 
double mutants, trypan blue staining was performed on wild type and mutant seedlings. As shown in Figure 5A, there was extensive cell death in the leaves of the $m k k 1$ $m k k 2$ double mutant plants, particularly along the veins. In contrast, cell death was not observed in the $m k k l$ and mkk2 single mutants. To check the $\mathrm{H}_{2} \mathrm{O}_{2}$ levels in the mutant plants, we performed DAB staining on the mutant seedlings. As shown in Figure 5B, strong staining was observed in the $m k k 1 m k k 2$ double mutants, but not in the wild-type and the single mutants, indicating that the $m k k 1 m k k 2$ mutant seedlings accumulated high levels of $\mathrm{H}_{2} \mathrm{O}_{2}$.

\section{Constitutive defense responses in $\mathrm{mkk} 1 \mathrm{mkk} 2$ plants}

To test whether defense responses were constitutively activated, the expression of $P R$ genes in the $m k k 1, m k k 2$ and $m k k 1 m k k 2$ mutant plants was determined by realtime RT-PCR. As shown in Figure 6A and 6B, high levels of $P R-1$ and $P R-2$ were constitutively expressed in the $m k k 1 m k k 2$ double mutant plants. In contrast, $P R-1$ and $P R-2$ were not constitutively expressed in the wildtype and the single mutant plants, suggesting that loss of function of both $M K K 1$ and $M K K 2$ leads to constitutive activation of defense responses.

To test whether the $m k k 1$ and $m k k 2$ single mutants and the $m k k 1 m k k 2$ double mutants have enhanced pathogen resistance, mutant seedlings were challenged with the virulent oomycete pathogen $H$. $p$. Noco2. As shown in Figure 6C, the $m k k 1 m k k 2$ double mutant seedlings showed strong enhanced resistance against $H$. $p$. Noco2 compared with the wild-type and the single mutants, confirming that defense responses were constitutively activated in the $m k k 1 m k k 2$ mutant plants.

\section{MKK1 and MKK2 are required for MPK4 activation}

MEKK1 has previously been shown to be required for the activation of MPK4 in response to the treatment of the flagellin peptide flg22. To test whether MKK1 and MKK2 are also required for the activation of MPK4 in response to flg22, we analyzed the kinase activity of immunoprecipitated MPK4 from wild-type and mutant plants. Myelin-basic protein was used as substrate for the assay. As shown in Figure 7A, MPK4 kinase activity was at very low levels when the plants were not treated with flg22. Spraying with flg22 resulted in quick activation of MPK4 in the wild-type plants. Whereas the activation of MPK4 was not affected in the single mutants of $m k k 1$ and $m k k 2$, activation of MPK4 was severely compromised in the mkk1-1 mkk2-1 double mutant, suggesting that MKK1 and MKK2 are required for the activation of MPK4 by flg22. Previously Meszaros et al. [17] showed that activation of MPK4 was blocked in $m k k 1$ single

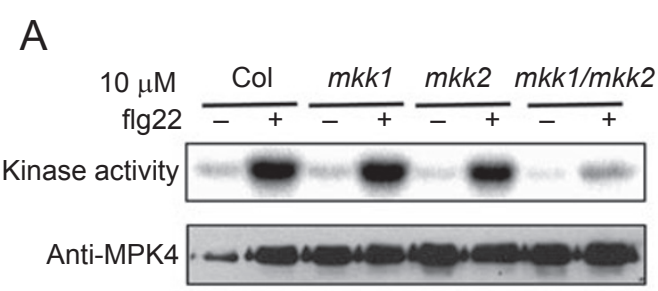

B

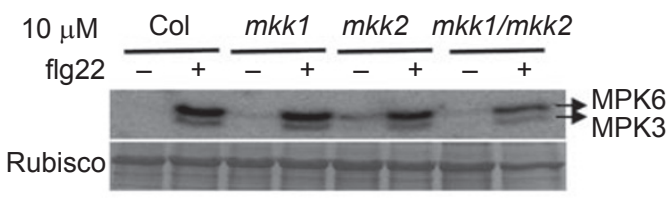

Figure 7 MAPK kinase activities in wild-type, $m k k 1, m k k 2$ and $m k k 1$ mkk2 double mutants. (A) Analysis of MPK4 activity upon flg22 treatment by immunocomplex kinase assay. Top panel: MPK4 kinase activity. Bottom panel: MPK4 protein levels detected by immunoblot analysis. (B) Analysis of MPK3 and MPK6 activity upon flg22 treatment by in-gel kinase assay. In both (A) and (B), 2-week-old seedlings were grown on 1/2 MS medium at $22{ }^{\circ} \mathrm{C}$. Seedlings were treated with $10 \mu \mathrm{M}$ flg22 for $15 \mathrm{~min}$ and subsequently collected in liquid nitrogen. Immunocomplex kinase assays were performed as described in the experimental procedures. In-gel kinase assays were performed as described previously [28].

knockout mutant plants. A recent report by Qiu et al. [18] found that MPK4 activity was not affected in $m k k 1$ single mutant plants, which is consistent with our findings. As Meszaros et al. used $1 \mu \mathrm{M}$ flg22 while Qiu et al. used $10 \mu \mathrm{M}$ flg22 for induction, the difference in MPK4 activation is probably because of the different concentrations of flg22 used.

In addition to MPK4, MPK3 and MPK6 were activated upon flg22 treatment [19]. To test whether MKK1 and MKK2 are required for the activation of MPK3 and MPK6, we used an in-gel kinase assay with myelin basic protein as the imbedded kinase substrate to measure the activity of these two kinases in the wild-type and mutant plants. As shown in Figure 7B, activation of MPK3 and MPK6 is not affected in either the $m k k 1$ and $m k k 2$ single mutants or the $m k k 1-1 m k k 2-1$ double mutant, suggesting that MKK1 and MKK2 are not required for the activation of MPK3 and MPK6 by flg22. In a recent report, Qiu et $a l$. found that activation of MPK3 and MPK6 by flg22 was compromised in the $m k k 1$ and $m k k 2$ single mutant and double mutant plants. We used in-gel kinase assays, whereas Qiu et al. performed kinase assays on immunoprecipitated MPK3 and MPK6. The difference in MPK3 and MPK6 activities observed in these two studies might 
be because of the different assays used.

\section{Discussion}

Arabidopsis MEKK1 is a negative regulator of cell death and defense responses. Knockout mutants of $M E K K 1$ displayed a seedling-lethality phenotype [1012]. MPK4 has been suggested to be the MAP kinase downstream of MEKK1 because activation of MPK4 by flg22 or $\mathrm{H}_{2} \mathrm{O}_{2}$ is impaired in the mekk1 mutants [1012]. However, the previously reported mpk4-1 mutant plants do not exhibit cell death and seedling-lethality phenotypes that were observed in the mekkl mutants [9]. Whereas mekkl mutants are in the Columbia backgound, $m p k 4-1$ is in the Landsberg background. The phenotypic difference between mpk4-1 and mekk1 mutants could be because of the genetic background difference. Here we report the identification of two new alleles of $m p k 4$ (mpk4-3 and mpk4-4) in the Columbia background. Like the mekk1 mutants, mpk4-3 and mpk4-4 exhibit spontaneous cell death and seedling-lethality phenotypes, suggesting that MPK4 and MEKK1 may function in the same MAPK kinase cascade.

MAP kinase cascades consist of three sequentially activated kinases, a MAP kinase kinase kinase (MAPKKK), a MAP kinase kinase (MAPKK) and a MAP kinase [20]. Our data suggest that MKK1 and MKK2 are the MAPKKs that function together with MEKK1 and MPK4. MKK1 and MKK2 interact with both MEKK1 and MPK4 in vivo, and $m k k 1 m k k 2$ double knockout mutants display similar phenotypes as the mekk1 and $m p k 4$ mutants. Furthermore, activation of MPK4 by flg22 is severely compromised in $m k k 1 m k k 2$ double mutant plants, suggesting that MEKK1, MKK1/MKK2 and MPK4 form a MAP kinase cascade. Similar results were also reported by Qiu et al. [18]. As MPK4 is required for normal plant growth in the absence of external signals, one of the functions of the MEKK1, MKK1/MKK2 and MPK4 kinase cascade is most likely to perceive an endogenous plant signal for suppressing spontaneous cell death and defense activation.

Interestingly, interaction between MEKK1 and MKK1 or MEKK1 and MKK2 occurs mainly on the plasma membrane, whereas interaction between MPK4 and MKK1 or MPK4 and MKK2 occurs both on the plasma membrane and in the nucleus, suggesting that perception of the upstream signal occurs initially at the plasma membrane by MEKK1 and is subsequently transduced to the nucleus by the downstream kinases MKK1/MKK2 and MPK4.

One question is how disruption of the MEKK1, MKK1/MKK2 and MPK4 cascade leads to cell death and activation of defense responses. As mekk1, mkk1 mkk2 and mpk4 mutants all accumulate high levels of $\mathrm{H}_{2} \mathrm{O}_{2}$, and $\mathrm{H}_{2} \mathrm{O}_{2}$ is a known inducer of cell death in plants [21], over-accumulation of $\mathrm{H}_{2} \mathrm{O}_{2}$ is probably the cause of cell death in these mutants. $\mathrm{H}_{2} \mathrm{O}_{2}$ is usually produced when plants are under stress conditions. It is important to keep $\mathrm{H}_{2} \mathrm{O}_{2}$ levels under control to avoid its detrimental effects on plants. The MEKK1, MKK1/MKK2 and MPK4 cascade could be a major mechanism for down-regulating $\mathrm{H}_{2} \mathrm{O}_{2}$ levels in plants. Disruptions of this MAPK kinase cascade lead to over-accumulation of $\mathrm{H}_{2} \mathrm{O}_{2}$ and, subsequently, cell death. Downstream of the MAPK kinase cascade, MPK4 has been shown to interact with MKS and phosphorylate two MKS-associated WRKY transcription factors, WRKY25 and WRKY33, in vitro [22]. It remains to be determined whether WRKY25 and WRKY33 also function in the regulation of $\mathrm{H}_{2} \mathrm{O}_{2}$ levels.

The cell death and constitutive activation of defense responses in the mekk1, mpk4 and $m k k 1 m k k 2$ double mutants are very similar to those of the mutant phenotypes caused by auto-activation of $\mathrm{R}$ proteins. Many $\mathrm{R}$ protein-mediated defense responses are also suppressed at high temperatures, suggesting a potential link between the MEKK1, MKK1/MKK2 and MPK4 kinase cascade and $\mathrm{R}$ protein-dependent defense pathways. However, it is unclear whether the high $\mathrm{H}_{2} \mathrm{O}_{2}$ levels in the mekkl, $m p k 4$ and $m k k 1 m k k 2$ mutants are the cause or result of activation of $\mathrm{R}$ protein-mediated signaling pathways.

Arabidopsis MPK4 is activated under a variety of biotic and abiotic stress conditions $[15,23]$, probably through the MEKK1, MKK1/MKK2 and MPK4 kinase cascade. How different stresses activate the same downstream MAPK kinase cascade is unclear. One possibility is that a common signal is generated under different stress conditions. The signal is perceived by an unknown receptor upstream of MEKK1, which subsequently activates the MEKK1, MKK1/MKK2 and MPK4 kinase cascade. As $\mathrm{H}_{2} \mathrm{O}_{2}$ is produced under various stress conditions and it can activate MPK4 by itself, it is plausible to hypothesize that $\mathrm{H}_{2} \mathrm{O}_{2}$ serves as a general stress signal upstream of the MEKK1, MKK1/MKK2 and MPK4 kinase cascade in a negative feedback loop to down-regulate the $\mathrm{H}_{2} \mathrm{O}_{2}$ level. The mechanisms by which $\mathrm{H}_{2} \mathrm{O}_{2}$ activates its downstream cascade are yet to be determined by future studies.

\section{Material and Methods}

\section{Mutant screen and mapping}

To identify mutants that constitutively express $P R-2$ (BGL2), npr 1-1 plants that contain the $p B G L 2-G U S$ reporter gene were mutagenized with EMS. The progeny of individual M1 lines were grown on MS medium and GUS staining was performed on half 
of the seedlings from each line when they were 2 weeks old. Lines displaying constitutive GUS staining were recorded and the remaining half of the seedlings from these lines were transplanted to soil to set seeds. To identify mutants that are resistant to $H$. $p$. Noco2, the progeny of individual M1 lines were planted in soil. Two-week-old seedlings were sprayed with $H$. p . Noco2 at a concentration of 50000 spores per $\mathrm{ml}$ and scored 7 days after inoculation. Mutants that displayed seedling-lethality phenotypes were propagated with plants that are heterozygous for the mutation.

To map the mpk4 and mekkl mutations in the Col background, the mutants were crossed with wild type Ler plants. The F2 progeny homozygous for the mpk4 and mekkl mutations were identified by their morphology. The locations of the mutations were determined using In-del markers on different chromosomes.

\section{BiFC analysis}

The MEKK1, MKK1, MKK2 and MPK4 cDNA was amplified by PCR from Col total cDNA. The PCR products of MEKK1 and $M P K 4$ were cloned into pUC-SPYCE that contained the $35 \mathrm{~S}$ promoter and the C-terminal region of YFP to obtain pMEKK1-YCE and pMPK4-YCE. The PCR products of MKK1 and $M K K 2$ were cloned into pUC-SPYNE that contained the $35 \mathrm{~S}$ promoter and N-terminal region of YFP to obtain pMKK1-YNE and pMKK2YNE, respectively. All constructs were confirmed by sequencing. Transient expression using Arabidopsis mesophyll protoplasts was performed according to a procedure described previously [24].

\section{Mutant characterization}

Plants were grown at $22{ }^{\circ} \mathrm{C}$ in $16 \mathrm{~h} \mathrm{light} / 8 \mathrm{~h}$ darkness on soil or $1 / 2$ MS medium. $m k k 1-1$ was kindly provided by Dr Bögre [17]. $m k k 1-2$ (SALK_027645) and $m k k 2-1$ (SAIL_511_H01) were obtained from the ABRC. $m k k 1-1$ and $m k k 1-2$ were crossed with $m k k 2-1$ to generate the $m k k 1 m k k 2$ double mutants. Expression of $P R$ genes was analyzed as described previously [25]. Trypan blue and DAB staining were performed on 16-day-old seedlings grown on $1 / 2$ MS medium according to procedures described previously $[26,27]$.

For the H. $p$. Noco2 infection experiments, plants were kept at $18{ }^{\circ} \mathrm{C}$ in $12 \mathrm{~h} \mathrm{light} / 12 \mathrm{~h}$ dark cycles with $95 \%$ humidity after being sprayed with the spores of H. p. Noco2 at a concentration of 50000 spores per $\mathrm{ml}$ water.

\section{Kinase assays}

For MPK3, MPK4 and MPK6 kinase assay, 2-week-old seedlings grown on $1 / 2 \mathrm{MS}$ medium were treated with $10 \mu \mathrm{M}$ flg2 2 for $15 \mathrm{~min}$ and subsequently collected in liquid nitrogen. The plant tissues were ground and homogenized in extraction buffer containing $50 \mathrm{mM}$ HEPES-KOH, pH 7.4, $50 \mathrm{mM} \mathrm{NaCl}, 10 \mathrm{mM}$ EDTA, $5 \mathrm{mM}$ $\mathrm{NaF}, 1 \mathrm{mM} \mathrm{Na} \mathrm{VO}_{4}, 0.1 \%$ Triton-X 100, 1 mM DTT, $1 \mathrm{mM}$ PMSF and $1 \times$ protease inhibitor cocktail (Sigma). After centrifugation at $14000 \mathrm{rpm}$ for $15 \mathrm{~min}$ at $4{ }^{\circ} \mathrm{C}$, the protein concentration was determined using a Bio-Rad Bradford protein assay kit. An equal amount of total protein for each genotype was used in subsequent assay. For immuno-complex kinase assays, protein extracts were incubated with polyclonal antibodies of MPK4 (Sigma) for $1 \mathrm{~h}$ at $4{ }^{\circ} \mathrm{C}$, and $20 \mu \mathrm{l}$ protein A-agarose (GE) was subsequently added and incubated for another $3 \mathrm{~h}$ at $4{ }^{\circ} \mathrm{C}$. The kinase on the beads was collected by centrifugation and washed thrice with extraction buffer and once with kinase buffer (50 mM HEPES-KOH, pH 7.4, 1
mM DTT, $10 \mathrm{mM} \mathrm{MgCl} 2,10 \mathrm{mM} \mathrm{MnCl} 2$ and $10 \mu \mathrm{M}$ ATP). Kinase assays on the immunoprecipitated MPK4 were performed at room temperature for $30 \mathrm{~min}$ in $15 \mu \mathrm{l}$ of kinase buffer containing myelin basic protein (MBP) (Upstate) as the artificial substrate and 2.5 $\mu \mathrm{Ci}$ of $\left[\gamma^{32} \mathrm{P}\right] \mathrm{ATP}$. Reactions were ended by the addition of SDSPAGE loading buffer. Phosphorylation of MBP was analyzed by autoradiography after SDS-PAGE. The in-gel kinase activity assay was performed according to a procedure described previously [28] using $7 \mu \mathrm{g}$ of total protein.

\section{Acknowledgments}

We would like to thank Dr Dongtao Ren from the China Agricultural University for his helpful suggestions on in-gel kinase assays; Dr László Bögre from the Royal Holloway University of London for donating the seeds of $m k k 1-1$; and Dr Xin Li from the University of British Columbia for critically reading the manuscript. We are grateful for financial supports to YZ from the Chinese Ministry of Science and Technology.

\section{References}

1 Jones JD, Dangl JL. The plant immune system. Nature 2006; 444:323-329.

2 Gomez-Gomez L, Boller T. FLS2: an LRR receptor-like kinase involved in the perception of the bacterial elicitor flagellin in Arabidopsis. Mol Cell 2000; 5:1003-1011.

3 Zipfel C, Kunze G, Chinchilla D, et al. Perception of the bacterial PAMP EF-Tu by the receptor EFR restricts Agrobacterium-mediated transformation. Cell 2006; 125:749-760.

4 Shirano Y, Kachroo P, Shah J, Klessig DF. A gain-of-function mutation in an Arabidopsis Toll Interleukin1 receptor-nucleotide binding site-leucine-rich repeat type $\mathrm{R}$ gene triggers defense responses and results in enhanced disease resistance. Plant Cell 2002; 14:3149-3162.

5 Zhang Y, Goritschnig S, Dong X, Li X. A gain-of-function mutation in a plant disease resistance gene leads to constitutive activation of downstream signal transduction pathways in suppressor of npr1-1, constitutive 1. Plant Cell 2003; 15:26362646.

6 Noutoshi Y, Ito T, Seki M, et al. A single amino acid insertion in the WRKY domain of the Arabidopsis TIR-NBS-LRRWRKY-type disease resistance protein SLH1 (sensitive to low humidity 1) causes activation of defense responses and hypersensitive cell death. Plant $J$ 2005; 43:873-888.

7 Igari K, Endo S, Hibara K, et al. Constitutive activation of a CC-NB-LRR protein alters morphogenesis through the cytokinin pathway in Arabidopsis. Plant J 2008; 55:14-27.

8 Pedley KF, Martin GB. Role of mitogen-activated protein kinases in plant immunity. Curr Opin Plant Biol 2005; 8:541-547.

9 Petersen M, Brodersen P, Naested H, et al. Arabidopsis map kinase 4 negatively regulates systemic acquired resistance. Cell 2000; 103:1111-1120.

10 Ichimura K, Casais C, Peck SC, Shinozaki K, Shirasu K. MEKK1 is required for MPK4 activation and regulates tissuespecific and temperature-dependent cell death in Arabidopsis. 
J Biol Chem 2006; 281:36969-36976.

11 Nakagami H, Soukupova H, Schikora A, Zarsky V, Hirt H. A Mitogen-activated protein kinase kinase kinase mediates reactive oxygen species homeostasis in Arabidopsis. J Biol Chem 2006; 281:38697-38704.

12 Suarez-Rodriguez MC, Adams-Phillips L, Liu Y, et al. MEKK1 is required for flg22-induced MPK4 activation in Arabidopsis plants. Plant Physiol 2007; 143:661-669.

13 Ichimura K, Mizoguchi T, Irie K, et al. Isolation of ATMEKK1 (a MAP kinase kinase kinase)-interacting proteins and analysis of a MAP kinase cascade in Arabidopsis. Biochem Biophys Res Commun 1998; 253:532-543.

14 Mizoguchi T, Ichimura K, Irie K, et al. Identification of a possible MAP kinase cascade in Arabidopsis thaliana based on pairwise yeast two-hybrid analysis and functional complementation tests of yeast mutants. FEBS Lett 1998; 437:56-60.

15 Teige M, Scheikl E, Eulgem T, et al. The MKK2 pathway mediates cold and salt stress signaling in Arabidopsis. Mol Cell 2004; 15:141-152.

16 Walter M, Chaban C, Schutze K, et al. Visualization of protein interactions in living plant cells using bimolecular fluorescence complementation. Plant J 2004; 40:428-438.

17 Meszaros T, Helfer A, Hatzimasoura E, et al. The Arabidopsis MAP kinase kinase MKK1 participates in defence responses to the bacterial elicitor flagellin. Plant J 2006; 48:485-498.

18 Qiu JL, Zhou L, Yun BW, et al. Arabidopsis MAP kinase kinases MKK1 and MKK2 have overlapping functions in defense signalling mediated by MEKK1, MPK4 and MKS1. Plant Physiol 2008; 148:212-222.

19 Asai T, Tena G, Plotnikova J, et al. MAP kinase signalling cascade in Arabidopsis innate immunity. Nature 2002; 415:977-
983.

20 MAPK-Group. Mitogen-activated protein kinase cascades in plants: a new nomenclature. Trends Plant Sci 2002; 7:301-308.

21 Levine A, Tenhaken R, Dixon R, Lamb C. H2O2 from the oxidative burst orchestrates the plant hypersensitive disease resistance response. Cell 1994; 79:583-593.

22 Andreasson E, Jenkins T, Brodersen P, et al. The MAP kinase substrate MKS1 is a regulator of plant defense responses. EMBO J 2005; 24:2579-2589.

23 Droillard MJ, Boudsocq M, Barbier-Brygoo H, Lauriere C. Involvement of MPK4 in osmotic stress response pathways in cell suspensions and plantlets of Arabidopsis thaliana: activation by hypoosmolarity and negative role in hyperosmolarity tolerance. FEBS Lett 2004; 574:42-48.

24 Sheen J. Signal transduction in maize and Arabidopsis mesophyll protoplasts. Plant Physiol 2001; 127:1466-1475.

25 Zhang Y, Tessaro MJ, Lassner M, Li X. Knockout analysis of Arabidopsis transcription factors TGA2, TGA5, and TGA6 reveals their redundant and essential roles in systemic acquired resistance. Plant Cell 2003; 15:2647-2653.

26 Parker JE, Holub EB, Frost LN, Falk A, Gunn ND, Daniels MJ. Characterization of eds1, a mutation in Arabidopsis suppressing resistance to Peronospora parasitica specified by several different RPP genes. Plant Cell 1996; 8:2033-2046.

27 Thordal-Christensen H, Zhang Z, Wei Y, Collinge DB. Subcellular localization of $\mathrm{H}_{2} \mathrm{O}_{2}$ in plants. $\mathrm{H}_{2} \mathrm{O}_{2}$ accumulation in papillae and hypersensitive response during the barley-powdery mildew interaction. Plant J 1997; 11:1187-1194.

28 Ren D, Yang H, Zhang S. Cell death mediated by MAPK is associated with hydrogen peroxide production in Arabidopsis. $J$ Biol Chem 2002; 277:559-565. 